$7-2015$

\title{
Quantification of Periodic Breathing in Premature Infants
}

\author{
Mary A. Mohr \\ William \& Mary \\ Karen D. Fairchild \\ Manisha Patel \\ et al. \\ John B. Delos \\ William \& Mary
}

Follow this and additional works at: https://scholarworks.wm.edu/aspubs

\section{Recommended Citation}

Mohr, Mary A.; Fairchild, Karen D.; Patel, Manisha; al., et; and Delos, John B., Quantification of Periodic Breathing in Premature Infants (2015). Physiological Measurement, 36(7), 1415-1427. https://doi.org/10.1088/0967-3334/36/7/1415

This Article is brought to you for free and open access by the Arts and Sciences at W\&M ScholarWorks. It has been accepted for inclusion in Arts \& Sciences Articles by an authorized administrator of W\&M ScholarWorks. For more information, please contact scholarworks@wm.edu. 


\title{
Quantification of periodic breathing in premature infants
}

\author{
Mary A Mohr ${ }^{1}$, Karen D Fairchild ${ }^{2}$, Manisha Patel ${ }^{2}$, \\ Robert A Sinkin ${ }^{2}$, Matthew T Clark ${ }^{3}$, J Randall Moorman ${ }^{3,4,5}$, \\ Douglas E Lake ${ }^{3,6}$, John Kattwinkel ${ }^{2}$, John B Delos ${ }^{1}$ \\ ${ }^{1}$ Department of Physics, College of William and Mary, Williamsburg, \\ VA 23187-8795, USA \\ 2 Department of Pediatrics (Neonatology), University of Virginia, Charlottesville, \\ Virginia \\ 3 Division of Cardiovascular Medicine, University of Virginia, Charlottesville, Virginia \\ ${ }^{4}$ Department of Biomedical Engineering, University of Virginia, Charlottesville, \\ Virginia \\ ${ }^{5}$ Department of Molecular Physiology, University of Virginia, Charlottesville, Virginia \\ ${ }^{6}$ Department of Statistics, University of Virginia, Charlottesville, Virginia
}

E-mail:mmohr@email.wm.edu

Received 29 December 2014, revised 26 February 2015

Accepted for publication 16 March 2015

Published 27 May 2015

\begin{abstract}
Periodic breathing $(\mathrm{PB})$, regular cycles of short apneic pauses and breaths, is common in newborn infants. To characterize normal and potentially pathologic $\mathrm{PB}$, we used our automated apnea detection system and developed a novel method for quantifying PB. We identified a preterm infant who died of sudden infant death syndrome (SIDS) and who, on review of her breathing pattern while in the neonatal intensive care unit (NICU), had exaggerated PB.

We analyzed the chest impedance signal for short apneic pauses and developed a wavelet transform method to identify repetitive $10-40$ second cycles of apnea/breathing. Clinical validation was performed to distinguish PB from apnea clusters and determine the wavelet coefficient cutoff having optimum diagnostic utility. We applied this method to analyze the chest impedance signals throughout the entire NICU stays of all 70 infants born at 32 weeks' gestation admitted over a two-and-a-half year period. This group includes an infant who died of SIDS and her twin.

For infants of 32 weeks' gestation, the fraction of time spent in PB peaks $7-14 \mathrm{~d}$ after birth at $6.5 \%$. During that time the infant that died of SIDS spent $40 \%$ of each day in PB and her twin spent $15 \%$ of each day in PB.

This wavelet transform method allows quantification of normal and potentially pathologic PB in NICU patients.
\end{abstract}


Keywords: periodic breathing, respiration, SIDS

(Some figures may appear in colour only in the online journal)

\section{Introduction}

Periodic breathing (PB), characterized by regular, repeated cycles of apneic pauses and breathing, has generally been considered to be a normal respiratory pattern in most if not all newborn infants (Rigatto 2003).However, several decades ago, excessive amounts of PB were observed in infants who had 'near-miss sudden infant death syndrome (SIDS)' and in siblings of infants that died of SIDS (Kelly and Shannon 1979, Kelly et al 1980). A recent case of SIDS in a preterm infant discharged home from the University of Virginia (UVa) Neonatal Intensive Care Unit (NICU) led to an unexpected finding: on retrospective review of our research database, we found that this infant spent a strikingly large proportion of time in PB compared to other preterm infants, despite having almost no episodes of classical apnea of prematurity (AOP). To our knowledge this is the first time that excessive PB has been observed in an NICU patient who subsequently died of SIDS. We hypothesize that exaggerated PB can be pathologic in newborn infants. As a first step toward testing this hypothesis, we report here the development of a new method of identifying and quantifying PB.

We want to detect periodic breathing and distinguish it from other types of abnormal respiration. A widely accepted definition of PB has at least 3 cyclical apneas of at least $3 \mathrm{~s}$ duration with less than $20 \mathrm{~s}$ of breathing in between (Barrington and Finer 1990). As we will show, our detector recognizes such events, but we have reason to believe that this definition is too broad to provide a useful warning of impending pathology. First, we and others have observed that such brief episodes are very common, even in the absence of any indications of pathology. Second, physiological models of PB contain a natural distinction between transient oscillations and sustained oscillations. According to these models, PB represents high gain in the control loop (Milhorn and Guyton 1965, Khoo et al 1982, Rapoport et al 1993, Takahashi and Doi 1993, Tehrani 1997, Batzel and Tran 2000, Berger et al 2000, Fowler and Kalamangalam 2000, Levine et al 2004, Cherniack et al 2005, Norman et al 2006, Verma et al 2009, Hall and Guyton 2010, Ben-Tal and Smith 2010). In neonates, high 'gain' appears to result from hypersensitivity of the chemoreceptors that trigger breaths in response to changes in blood gases (Al-Matary et al 2004, Cherniack and Longobardo 2006, Edwards et al 2013). Peripheral chemoreceptors are desensitized at birth, with the acute increase in blood oxygen content during fetal to neonatal transition, then are gradually reset by about one week of age at which time PB emerges (Barrington and Finer 1990). Hypersensitivity of chemoreceptors to changes in blood oxygen and carbon dioxide levels leads to self-sustained oscillations between breathing and apneic pauses, especially during quiet sleep (Pereira et al 1995, Rigatto 2003).

In contrast to newborn infants, healthy adults rarely exhibit significant $\mathrm{PB}$, except with acute exposure to hypoxia at high altitude (Fowler and Kalamangalam 2002, Ainslie et al 2013). Acute and chronic diseases can, however, lead to PB patterns such as Cheyne-Stokes respiration associated with heart failure (Lange and Hecht 1962, Dowell et al 1971, Lieber and Mohsenin 1992Vielle and Chauvet 1993a, 1993b, Francis et al 2000, Vielle and Chauvet 1998, Manisty et al 2006). Cheyne-Stokes respiration is characterized by a regular pattern of respiration and apnea; the cycle time in adults ranges from $30 \mathrm{~s}$ to two minutes, and during the respiratory phase, both the amplitude and the frequency of breathing wax and wane in a regular crescendo-decrescendo pattern. Heart failure may cause excessive time delay in the control loop, which can also lead to high loop gain, resulting in PB. A similar pattern was seen in a 
small number of stroke patients (Hermann et al 2007). A distinction between the patterns of neonatal PB and adult Cheyne-Stokes breathing is that the cycle time in the former may average around $15 \mathrm{~s}$, while in the latter it may average about a minute. Both cases are apparently associated with high loop gain, but the physiological causes may be distinct: chemoreceptor hypersensitivity in infants versus excessive time delay in heart failure.

There are several other types of abnormal respiration that are easily confused with periodic breathing, and the distinctions among them are not sharp. An excellent review is given by Richerson and Boron (Richerson and Boron 2005), who identify, besides CheyneStokes respiration, Biot breathing, ataxic breathing, cluster breathing and gasping. During a study of Cheyne-Stokes respiration, Biot (Wijdicks 2007) came across a distinct pattern in patients with meningitis: there were irregular pauses lasting 10-30 s, and the breathing was irregular and rapid, without the crescendo-decrescendo pattern. Similar to Biot breathing are patterns called cluster breathing or ataxic respiration, which can occur as a result of lesions in the pons or medulla. It appears that there are not precisely defined differences among these.

In summary, physiological models of periodic breathing involve a control system in which parameters have changed so that it has gone from a stable steady state to a stable limit cycle. For values of parameters between those leading to steady state and those leading to a limit cycle, one finds oscillatory decay to the steady state. In contrast, Biot breathing, cluster breathing, and ataxic breathing seem to involve more profound failure of the control system. To construct a pattern-recognition algorithm for periodic breathing, we focus not on the breathing, but on the apneas, and we ask: (1) are the apneas occurring with a regular rhythm? (2) Is that rhythm sustained, or does it stop after a short time?

\section{Methods}

\subsection{Patient population and data collection}

We collected and stored all waveform and vital sign data from NICU bedside monitors (GE Medical, models Solar 8000M and I and Dash 3000) via a BedMaster central network server (Excel Medical, Jupiter, FL) for all patients in the UVa NICU from January 2009 to June 2011. The UVa NICU is a quaternary care unit that admits approximately 500 newborns per year, about half of them preterm and a quarter very low birth weight $(<1500 \mathrm{~g})$. Chest impedance (CI) waveforms were collected at $60 \mathrm{~Hz}, 3$ channels of electrocardiogram at $240 \mathrm{~Hz}$, and pulse oximetry at $120 \mathrm{~Hz}$. Vital signs (heart rate, respiratory rate, and oxygen saturation) were collected every $2 \mathrm{~s}$. This research was approved by the Institutional Review Boards of the UVa School of Medicine and the College of William and Mary.

\subsection{Chest impedance waveform analysis}

Our research group recently advanced the study of neonatal apnea by developing an automated detection system that analyzes chest impedance waveforms from bedside monitors and identifies all cessations of chest movement (Lee et al 2012). The standard definition of apnea of prematurity (AOP) is either a cessation of breathing lasting at least $20 \mathrm{~s}$, or a cessation of breathing lasting at least $10 \mathrm{~s}$ when accompanied by bradycardia and oxygen (Finer et al 2006). The automated detection system filters out heartbeat and movement artifact to produce a filtered chest impedance signal, from which, at quarter second intervals, the probability of apnea signal (the probability that the infant is having a cessation of chest movement at that moment) is computed (figure 1). The thresholds we use are $>10 \mathrm{~s}$ 


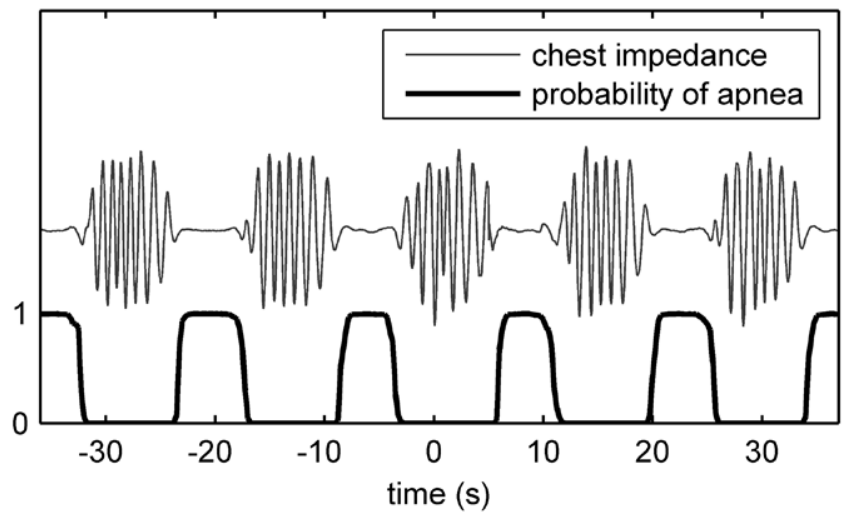

Figure 1. Periodic breathing in the chest impedance signal and the probability of apnea signal in a preterm infant that died of SIDS. The filtered chest impedance signal (top) and the computed probability of apnea signal (bottom) show $70 \mathrm{~s}$ of $\mathrm{PB}$, with cycles consisting of eight breaths followed by an apneic pause of about $6 \mathrm{~s}$. This example shows a typical duration of the PB cycle of about $15 \mathrm{~s}$, with more time spent in breathing than in apnea. Our wavelet transform method detects periodic breathing with cycle lengths from 10 to $40 \mathrm{~s}$, and apnea:breathing ratios of 1:4 to 4:1.

cessation of breathing, $\mathrm{HR}<100 \mathrm{bpm}$, and $\mathrm{SpO}_{2}<80 \%$; we call such events ABD10 (Lee et al 2012). This method needs to be modified for the present purposes because we observe that cessations of breathing associated with PB are typically 6-9s, and, while heart rate and oxygen saturation may sometimes be entrained with the breathing cycle, the reduction of heart rate and oxygen rarely reach the bradycardia or desaturation thresholds defined for clinical apnea.

Accordingly, for study of $\mathrm{PB}$, we take any cessation of breathing greater than $2 \mathrm{~s}$ to be an 'apneic pause' or an 'apnea', and we seek periodicity in these pauses. Our entire dataset includes 1437 infants, with 51.8 infant-years of analyzable data. From this set we selected infants of 32 weeks gestational age for closer study.

\subsection{Wavelet transform analysis}

PB was identified using a continuous wavelet transform (a type of time-frequency transformation) of the probability of apnea signal, without regard to heartrate or desaturation. The continuous wavelet transformation is a convolution of an input function with functions that are calculated from a mother wavelet.

Two mother wavelets, $\psi(t)$ with 6 cycles were designed for detecting PB. Using a sine window to weight the middle of the wavelets more heavily than the ends (figure 2(a)) allowed detection of PB with as few as 3 cycles. One mother wavelet has equal amounts of time in apnea (A) and breathing (B). A second mother wavelet has an A:B ratio of 1:2 (or 2:1). These wavelets are sufficient for detection of $\mathrm{PB}$ with $\mathrm{A}: \mathrm{B}$ ratios from 1:4 through 4:1, since the wavelet coefficient remains above the threshold value of 0.6 in this range.

A family of wavelets, $\psi_{s, \tau}(t)$, was generated by expanding or contracting the mother wavelets along the horizontal axis (figure 2(b)). This accounts for variability in the PB cycle duration from 10-40 s. PB is usually seen in the 10-20s range (Barrington et al 1987, Glotzbach et al 1989, Wilkinson et al 2007), but PB has been defined as having breathing segments of up to $20 \mathrm{~s}$ duration (Barrington and Finer 1990). PB was analyzed for each infant during the 


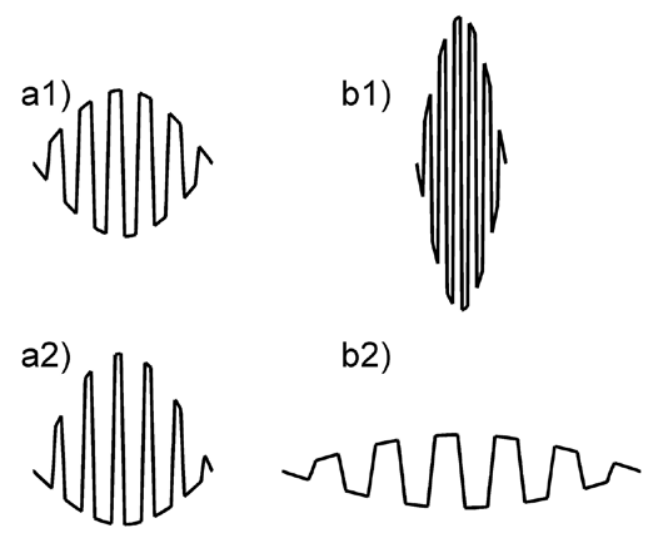

Figure 2. Mother wavelets and scaled wavelets for detecting PB. The two mother wavelets are modeled after the appearance of the probability of apnea signal during periodic breathing, with multiplication by a sine window to weight the middle of the wavelet more heavily. (a1) Mother wavelet with two phases (apnea and breathing) of equal duration. (a2) Mother wavelet with one phase twice as long as the other, created to detect a wide range of time ratios. $(b 1, b 2)$ Mother wavelets are scaled to different sizes (compressed or stretched along the horizontal axis), giving a family of wavelets that detect PB with cycle lengths ranging from 10-40 s.

entire NICU stay by shifting the family of wavelets along the apnea signal at quarter second intervals (figures $3(a)$ and $(b)$ ). The family of shifted and scaled wavelets is generated by $\psi_{s, \tau}(t)=|s|^{-p} \psi((t-\tau) / s)($ Kaiser 1994) where we have chosen $p=1 / 2$. For each scaled and shifted wavelet, a wavelet coefficient was calculated by taking the convolution (a measure of area overlap or of correlation) of the apnea signal and the wavelet (in general the complex conjugate of the wavelet, but in this case the wavelet is always real), $\gamma(s, \tau)=\int_{-\infty}^{\infty} f(t) \bar{\Psi}_{s, \tau}(t) \mathrm{d} t$. These coefficients, $\gamma(s, \tau)$ are a function of time and scale, indicating how closely repetitive apnea events 'match' the wavelet, normalized so that the maximum possible value is 1 . The wavelet coefficients rise and fall as the wavelet is shifted through an epoch of PB (figures 3(c) and $(d)$ ). The maximum value of the coefficients over $40 \mathrm{~s}$ from both mother wavelets across all scales was recorded every $20 \mathrm{~s}$. We chose to take the maximum value over $40 \mathrm{~s}$ because we are looking for PB with a cycle duration of up to 40 s. This value is the PB index. A preliminary analysis of the PB index suggested a threshold near 0.6 to distinguish PB. All times when this index was $\geq 0.6$ were marked as PB events.

\subsection{Clinical validation of the wavelet analysis}

For validation, 200 four-minute windows of bedside monitor data from all NICU patients, centered on an apnea event of at least $10 \mathrm{~s}$ ' duration were randomly selected by one author so that 100 had a PB index $\geq 0.6$ at any time and 100 did not. Of note, many of the windows with PB index $<0.6$ contained apnea clusters, as discussed below and as shown (figure 4). Four neonatologists independently evaluated each of the 200 windows of raw and filtered chest impedance, ECG, heart rate, and oxygen saturation, without knowledge of the criteria used for their selection. The neonatologists were asked to determine whether the breathing pattern fit the usual description of PB in neonates (at least 3 apnea/breathing cycles with cycle duration 10-40 s and a consistent rhythm). Events with less than $100 \%$ 
(a)

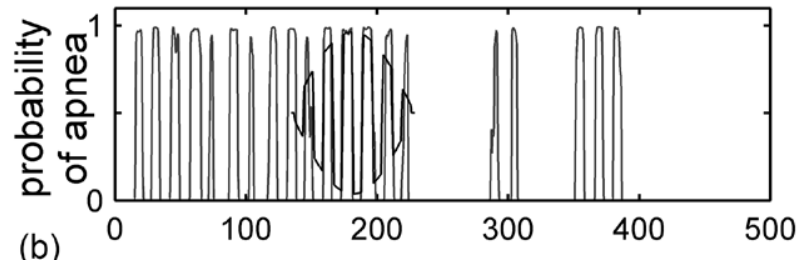

(b)

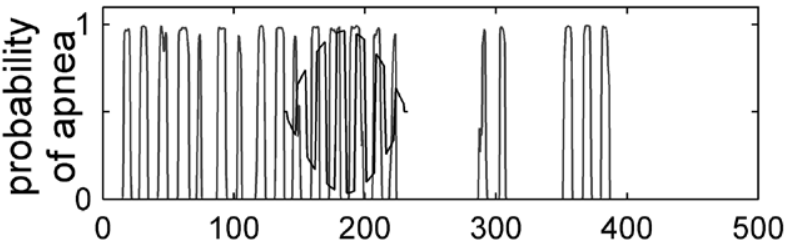

(c)

Wavelet Coefficients

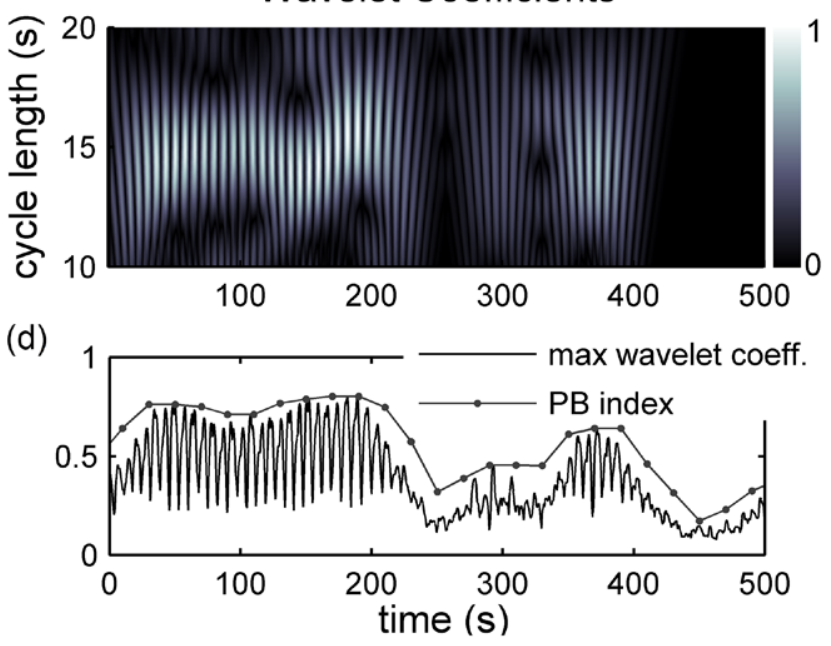

Figure 3. Wavelet transformation of probability of apnea signal to derive wavelet coefficients and PB index. $(a)$ and $(b) 500$ s window of the probability of apnea signal in a preterm infant, with shifted wavelet. At quarter second intervals, a wavelet coefficient is calculated that corresponds to the convolution of the wavelet and the probability of apnea signal. The absolute values of the coefficients range from 0 to 1 with a higher value indicating better alignment. PB is identified when the coefficient meets or exceeds the threshold value of 0.6. In $(a)$, the oscillations in the wavelet match those of the signal, giving a large wavelet coefficient, while in $(b)$, the oscillations are out of phase, and the coefficient is small. Thus the coefficients oscillate rapidly as the wavelet is translated through the signal. $(c)$ Wavelet coefficients for scales corresponding to cycle lengths from 10 to $20 \mathrm{~s}$. Lighter shades indicate higher values. $(d)$ The maximum of the absolute values of the wavelet coefficients across all scales at each point is shown along with the PB index, which is the maximum in a $40 \mathrm{~s}$ window calculated every $20 \mathrm{~s}$.

agreement in independent review by the 4 clinicians were subjected to group discussion, and consensus was reached in 180 cases. These 180 events were used to evaluate the predictive capabilities of our PB detection method. This wavelet method was used to quantify PB in all infants admitted during a 2.5 year time period. Below we discuss those of 32 week estimated gestational age (EGA), which includes the infant that died of SIDS after NICU discharge and her twin sister. 

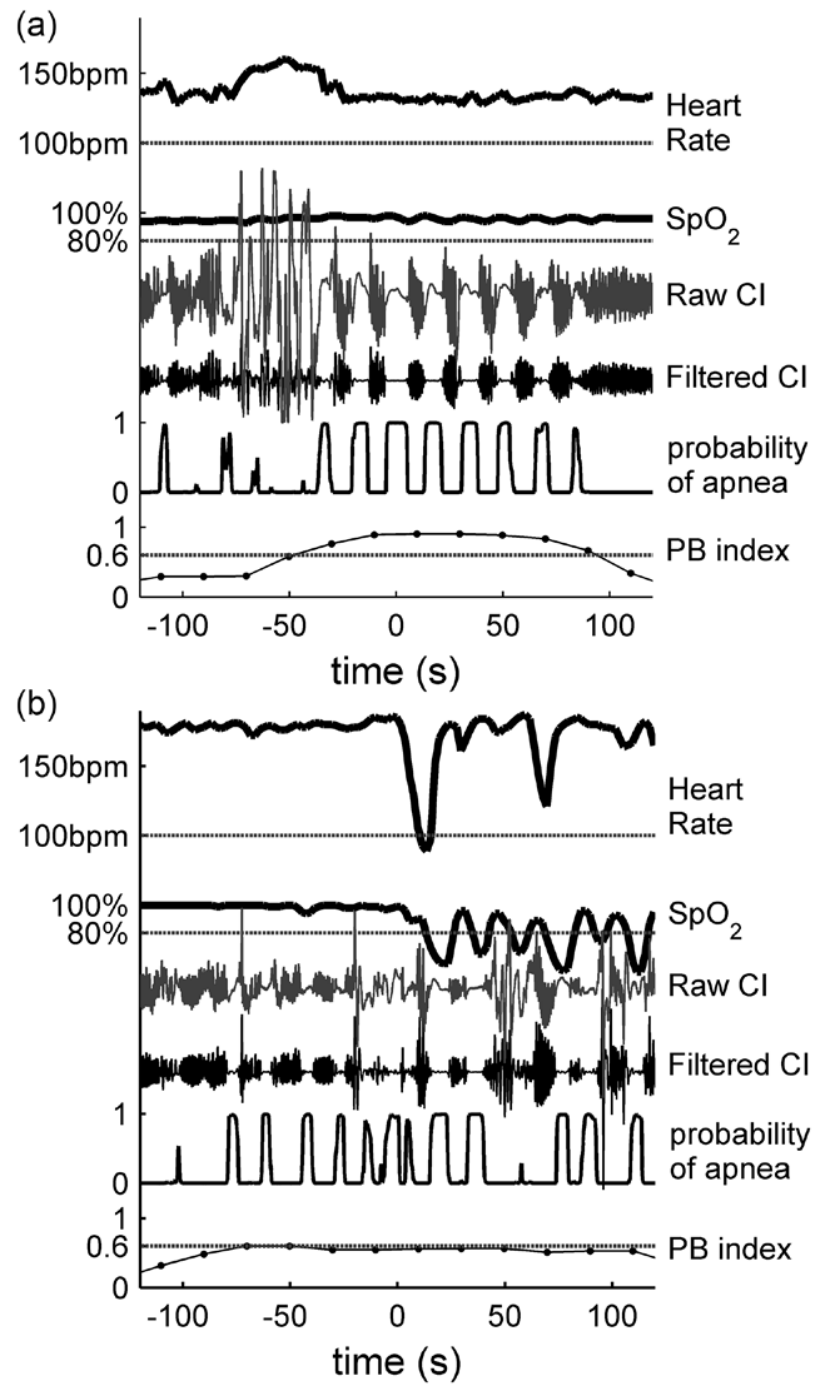

Figure 4. Periodic breathing versus clustered apnea. Four-minute window of heart rate, oxygen saturation $\left(\mathrm{SpO}_{2}\right)$, raw and filtered chest impedance $(\mathrm{CI})$, probability of apnea signal, and PB index. Periodic breathing is identified by the wavelet method when the PB index meets or exceeds the threshold value of 0.600. (a) Regular, repetitive apnea/ breathing oscillations (maximum wavelet coefficient $=0.905$ ) identifying true periodic breathing. (b) Cluster of irregular apnea/breathing. The computer did not identify this as definite PB since the wavelet coefficient was always below the threshold value ( maximum wavelet coefficient $=0.598$ ).

\section{Results}

\subsection{Clinical validation of the wavelet analysis}

The PB index for the 200 four-minute windows was calculated. Clinicians determined that 73 of the 200 windows included definite PB, 107 did not include PB, and 20 were disputed. Based on this result we tested the predictive capability of a periodic breathing index $\geq 0.6$ for 


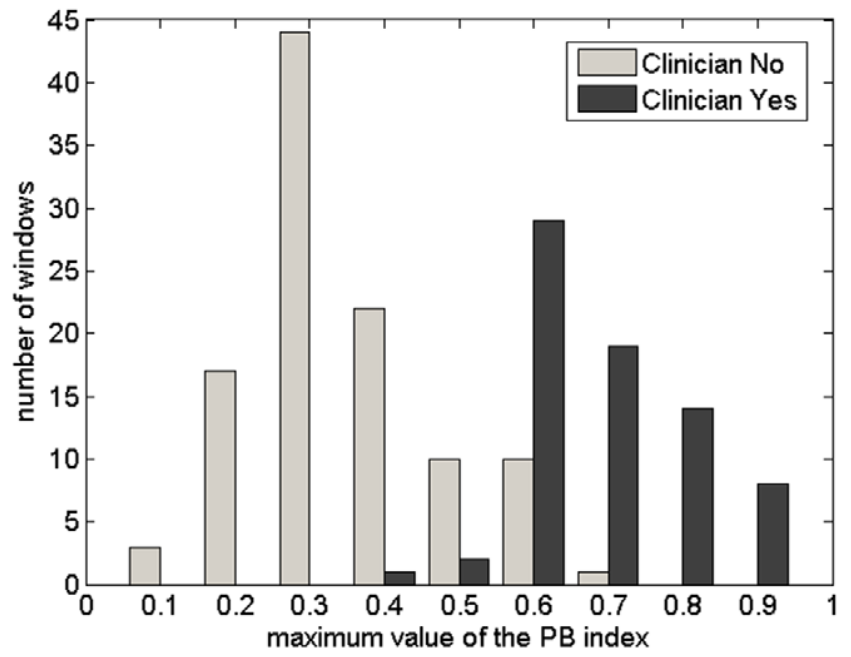

Figure 5. Superimposed histograms of the maximum value of the PB index. For the groups of clinician-identified PB (clinician yes) and no clinician identified PB (clinician no), the windows are classified according to the maximum value of the $\mathrm{PB}$ index in that window. The maximum PB index is at least as great as the value shown on the $x$-axis. (For example, there are 3 windows with a maximum $\mathrm{PB}$ index $\geq 0.1$ and $<0.2$.)

Table 1. Contingency table from validation of wavelet transform analysis.

\begin{tabular}{llllrl}
\hline & & \multicolumn{3}{c}{ Clinician } & \\
\cline { 3 - 5 } & & Yes & No & Total & \\
\hline Computer & Yes & 70 & 11 & 81 & Positive predictive value 86\% \\
& No & 3 & 96 & 99 & Negative predictive value 97\% \\
& Total & 73 & 107 & 180 & \\
& & Sensitivity & Specificity & & \\
& & $96 \%$ & $90 \%$ & & \\
\hline
\end{tabular}

detecting clinician-identified undisputed PB. The maximum values of the PB index in the four-minute windows were $0.74 \pm 0.03$ (mean $\pm 95 \% \mathrm{CI}$ ) for 73 windows with clinicianidentified undisputed PB, $0.40 \pm 0.02$ for 107 windows with no clinician-identified $\mathrm{PB}$, and $0.66 \pm 0.02$ for the 20 disputed windows (figure 5). Of the 73 windows that contained clinician-identified definite $\mathrm{PB}$, the computer-derived PB index was $\geq 0.6$ for 70 (96\% sensitivity). Other diagnostic utility measures are shown in table 1. Calculation of the ROC curve (area under the curve $=0.98$ ) showed that the product of sensitivity and specificity was maximized at the threshold of 0.6 , confirming the appropriateness of this value.

\section{2. $P B$ and $A O P$ in infants of 32 weeks' gestation}

In 32 weeks' gestation infants ( $n=70$, birthweight $1840 \pm 320 \mathrm{~g}$, length of NICU stay $26 \pm 12 \mathrm{~d}$ ) PB was generally not present in the first few days after birth, peaked between 1-2 weeks of age, and declined thereafter (figure 6). From day 7-14 after birth, the median percent of time spent in PB for all infants except the infant that died of SIDS and her twin was $3 \%$ (IQR $=9 \%$ ) (for $n=58$ patients for whom we have data during this week of life). In comparison, during the same time period, the average daily time in PB for the infant who died 


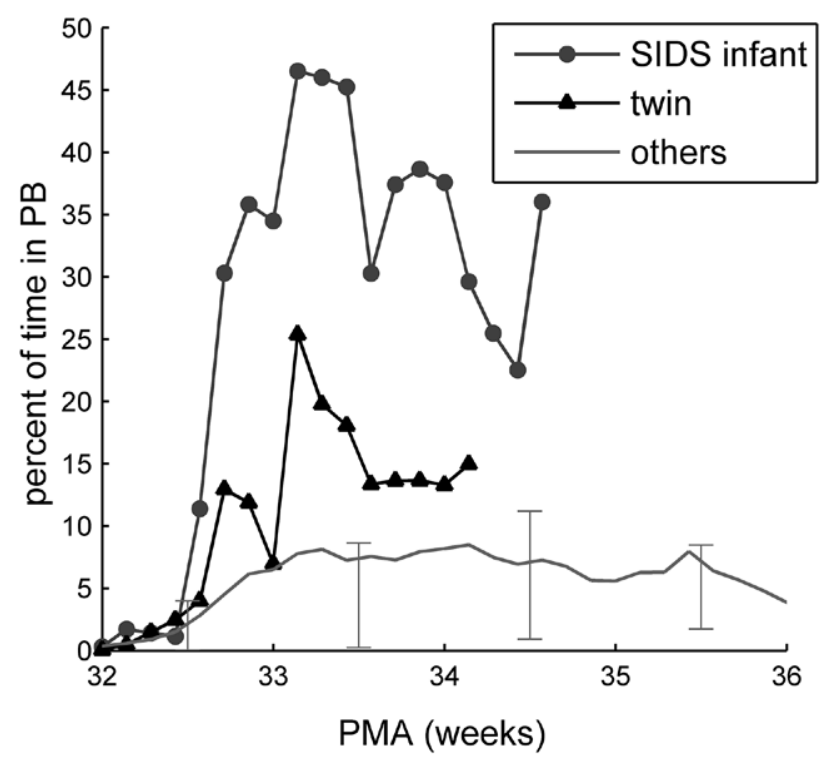

Figure 6. PB for SIDS infant and gestational age-matched infants. The percent of time spent in PB (time PB index $\geq 0.6 /$ time data available) is shown based on post-menstrual age (weeks) for the infant that died of SIDS 2 weeks after NICU discharge, her twin sister, and all other infants born at 32 weeks' gestation $(n=68)$. The 25 th and 75 th percentiles every week for all other infants of 32 weeks' gestation are indicated with error bars $(n=35-62)$

of SIDS was $40 \%$, and was still $36 \%$ at discharge. Her surviving twin had a daily average of $15 \% \mathrm{~PB}$ in the second week after birth (figure 6).

We also analyzed episodes of apnea accompanied by bradycardia and desaturation in these 32 weeks' gestation infants, using our previously published methods (Lee et al 2012). Remarkably, the SIDS infant and her twin each had only one computer-detected 'ABD' event during their 3 week NICU stay, defined as central apnea lasting at least $10 \mathrm{~s}$ associated with bradycardia $<100 \mathrm{bpm}$ and desaturation $<80 \%$. Neither of these events was documented in the medical record. The other 32 weeks' gestation infants had, on average, 31 computer-detected ABD events during the course of their NICU stay.

\section{Discussion}

Research on PB in infants has been limited, in part because this breathing pattern is transient and considered to be benign, and in part due to lack of methods to characterize and quantify PB in large numbers of infants over long periods of time. Our finding that a former NICU patient that died of SIDS had an inordinate amount of PB led to the development of an automated method to quantitate PB in all UVa NICU patients, a first step toward being able to quantify and characterize both normal and exaggerated, potentially pathologic PB in NICU patients.

\subsection{Distinguishing $P B$ from irregular clusters of $A O P$}

An important feature of our wavelet method is its ability to distinguish irregular clusters of AOP from PB. Two questions were asked: (1) Are the apnea events brief and occurring with 
a regular rhythm? (2) Is this regular apnea/breathing rhythm sustained for at least 3 cycles? Making this distinction is important. While PB can be described by physiological models with a well-behaved control system, in which the system can be in a stable steady state (normal respiration) or a stable limit cycle (PB) (Khoo et al 1982, Cherniack and Longobardo 2006), entirely different mechanisms may be responsible for clustered apnea. AOP and PB are distinct in character, gestational age predilection, and timing of onset and resolution (Barrington and Finer 1990). AOP is uncommon in infants $>34$ weeks' gestation, whereas PB is very common in both late preterm and term infants (Glotzbach et al 1989, Oliveira et al 2004). AOP begins within $1-2 \mathrm{~d}$ after birth and usually resolves between 36 and 42 weeks postmenstrual age (Eichenwald et al 1997), whereas PB typically starts after the first week and often persists beyond NICU discharge, for up to several months past term-corrected age (Wilkinson et al 1995). The timing of onset of PB coincides with the time that peripheral arterial chemoreceptors, silenced at birth with the acute rise in blood oxygen levels, become highly sensitive to small fluctuations in blood gases (Pereira et al 1995, Khan et al 2005).

PB typically includes apneic pauses of less than $10 \mathrm{~s}$ and may entrain only small or no decline in heart rate and oxygen saturation (Poets and Southall 1991, Razi et al 2002), rarely low enough to reach the threshold to trigger bedside monitor alarms, whereas AOP is often prolonged and associated with significant bradycardia and oxygen desaturation (Finer et al 2006). Hypoxia may trigger or exacerbate AOP or PB (Al-Matary et al 2004), and administering oxygen to preterm infants can minimize both (Weintraub et al 1992, Simakajornboon et al 2002).

\subsection{Exaggerated PB: a warning of pathology?}

In newborns, $\mathrm{PB}$ is considered normal, but our patient who died of SIDS spent a much greater proportion of time in PB than gestational and postmenstrual age-matched infants, clearly representing an abnormal breathing pattern. She had an uncomplicated NICU course, with no infections or respiratory disease, no AOP documented in the medical record, no caffeine treatment, and a normal head ultrasound. She was discharged at 3 weeks of age, was well at a visit to the pediatrician one week later, and was found dead in her crib two weeks after NICU discharge, at 37 weeks post-menstrual age. A death scene investigation and autopsy were performed and cause of death was determined to be SIDS. She had no evidence of neurologic, cardiovascular, or other pathology based on her clinical course in the NICU or on autopsy. Her twin sister also had exaggerated PB compared to other 32 weeks' gestation infants while in the NICU, (about two-fold as much compared to six-fold in the SIDS infant). One day after the death of her sister the surviving twin had an overnight sleep study that disclosed normal breathing patterns, and she subsequently underwent home cardiorespiratory monitoring for several months with no untoward events, and is currently 3 years old and healthy.

An association between exaggerated PB and SIDS risk was previously reported decades ago by Kelly and colleagues studying infants with 'near-miss SIDS' episodes (Kelly and Shannon 1979) and siblings of SIDS victims (Kelly et al 1980). The etiology of SIDS is multifactorial (Filiano and Kinney 1994, Kinney 2009, Duncan et al 2010, Jones 2012), and it is possible that our patient had other stressors after NICU discharge that led to terminal apnea. The relationship between PB and SIDS remains speculative, and further research is needed to determine to what extent quantitative or qualitative abnormalities of PB might be a sign of vulnerability to SIDS or a warning of other impending illnesses. Here, motivated by the observation of exaggerated PB in a case of SIDS, we have reported a method for quantitation of $\mathrm{PB}$. 


\subsection{Advantages of the method}

Past studies of PB have been limited to short monitoring times in small numbers of infants, often with visual inspection of respiratory impedance or inductance waveforms for characteristic apnea/breathing cycles. Our automated apnea algorithm gives a reliable measure of central apneic pauses, which can be used to quantify PB in large numbers of patients over long periods of time. This wavelet method distinguishes PB from apnea clusters where the durations of apnea and breathing are irregular (figure 4). With increasing availability of high-speed data processing it is likely that this method could ultimately be applied in near-real-time to assess for immature breathing patterns that may have important clinical implications.

\section{Acknowledgments}

This work was funded by NICHD grant 5RC2HD064488 (JRM, JK), and by NSF grant 1068344 (JBD). Computations were performed using facilities at The College of William and Mary, which were provided with the assistance of the National Science Foundation, the Virginia Port Authority, Sun Microsystems, and Virginia's Commonwealth Technology Research Fund.

\section{Competing interests}

JBD has a shared patent on the automated apnea detector.

\section{References}

Ainslie P N, Lucas S J and Burgess K R 2013 Breathing and sleep at high altitude, Respir. Physiol. Neurobiol. 188 233-56

Al-Matary A, Kutbi I, Qurashi M, Khalil M, Alvaro R, Kwiatkowski K, Cates D and Rigatto H 2004 Increased peripheral chemoreceptor activity may be critical in destabilizing breathing in neonates Semin. Perinatol. 28 264-72

Barrington K J and Finer N N 1990 Periodic breathing and apnea in preterm infants Pediatr. Res. $27118-21$

Barrington K J, Finer N N and Wilkinson M H 1987 Progressive shortening of the periodic breathing cycle duration in normal infants Pediatr. Res. $21247-51$

Batzel J and Tran H 2000 Modeling instability in the control system for human respiration: applications to infant non-REM sleep Appl. Math. Comput. 110 1-52

Ben-Tal A and Smith J C 2010 Control of breathing: two types of delays studied in an integrated model of the respiratory system Respir. Physiol. Neurobiol. 170 103-12

Berger K I, Ayappa I, Sorkin I B, Norman R G, Rapoport D M and Goldring R M $2000 \mathrm{CO}_{2}$ homeostasis during periodic breathing in obstructive sleep apnea J. Appl. Physiol. 88257

Cherniack N S and Longobardo G S 2006 Mathematical models of periodic breathing and their usefulness in understanding cardiovascular and respiratory disorders Exp. Physiol. 91 295-305

Cherniack N S, Longobardo G and Evangelista C J 2005 Causes of Cheyne-Stokes respiration Neurocritical Care 3 271-9

Duncan J R, Paterson D S, Hoffman J M, Mokler D J, Borenstein N S, Belliveau R A, Krous H F, Haas E A, Stanley C and Nattie E E 2010 Brainstem serotonergic deficiency in sudden infant death syndrome JAMA 303 430-7

Dowell A R, Buckley C E III, Cohen R, Whalen R E and Sieker H O 1971 Cheyne-Stokes respiration: a review of clinical manifestations and critique of physiological mechanisms Arch. Intern. Med. 127712

Edwards B A, Sands S A and Berger P J 2013 Postnatal maturation of breathing stability and loop gain: the role of carotid chemoreceptor development Respir. Physiol. Neurobiol. 185 144-55

Eichenwald E C, Aina A and Stark A R 1997 Apnea frequently persists beyond term gestation in infants delivered at 24-28 weeks Pediatrics 100 354-9 
Filiano J and Kinney H 1994 A perspective on neuropathologic findings in victims of the sudden infant death syndrome: the triple-risk model Neonatology 65 194-7

Finer N N, Higgins R, Kattwinkel J and Martin R J 2006 Summary proceedings from the apnea-ofprematurity group Pediatrics 117 S47-51

Fowler A C and Kalamangalam G P 2002 Periodic breathing at high altitude Math. Med. Biol. 19293

Fowler A C and Kalamangalam G P 2000 The role of the central chemoreceptor in causing periodic breathing IMA J. Math. Control Inf. 17147

Francis D P, Willson K, Davies L C, Coats A J S and Piepoli M 2000 Quantitative general theory for periodic breathing in chronic heart failure and its clinical implications Circulation 102 2214-21

Glotzbach S, Tansey P, Baldwin R and Ariagno R 1989 Periodic breathing cycle duration in preterm infants Pediatr. Res. 25 258-61

Hall J E and Guyton A C 2010 Textbook of Medical Physiology: Enhanced E-book (London: Elsevier Health Sciences)

Hermann D M, Siccoli M, Kirov P, Gugger M and Bassetti C L 2007 Central periodic breathing during sleep in acute ischemic stroke Stroke; J. Cerebral Circ. 38 1082-4

Jones S F 2012 SIDS and other sleep-related infant deaths: expansion of recommendations for a safe infant sleeping environment Year Book of Pulmonary Diseases ed J Barker (Philadelphia: Mosby Elsevier) pp 197

Kaiser G 1994 A Friendly Guide to Wavelets (Basel: Birkhäuser)

Kelly D H and Shannon D C 1979 Periodic breathing in infants with near-miss sudden infant death syndrome Pediatrics 63 355-60

Kelly D H, Walker A M, Cahen L and Shannon D C 1980 Periodic breathing in siblings of sudden infant death syndrome victims Pediatrics 66 515-20

Khan A, Qurashi M, Kwiatkowski K, Cates D and Rigatto H 2005 Measurement of the $\mathrm{CO}_{2}$ apneic threshold in newborn infants: possible relevance for periodic breathing and apnea J. Appl. Physiol. 98 1171-6 (Bethesda, MD, 1985)

Khoo M, Kronauer R E, Strohl K P and Slutsky A S 1982 Factors inducing periodic breathing in humans: a general model J. Appl. Physiol. 53 644-59

Kinney H C 2009 Brainstem mechanisms underlying the sudden infant death syndrome: evidence from human pathologic studies Dev. Psychobiol. $51223-33$

Lange R L and Hecht H H 1962 The mechanism of Cheyne-Stokes respiration J. Clin. Invest. 4142

Lee $\mathrm{H}$ et al 2012 A new algorithm for detecting central apnea in neonates Physiol. Meas. 33 1-17

Levine M, Hathorn M and Cleave J 2004 Mathematical methods in the study of respiration in newborn babies Cardiovasc. Eng. 4 133-8

Lieber C and Mohsenin V 1992 Cheyne-Stokes respiration in congestive heart failure Yale J. Biol. Med. $6539-50$

Manisty C H, Willson K, Wensel R, Whinnett Z I, Davies J E, Oldfield W L G, Mayet J and Francis D P 2006 Development of respiratory control instability in heart failure: a novel approach to dissect the pathophysiological mechanisms J. Physiol. 577387

Milhorn H T and Guyton A C 1965 An analog computer analysis of Cheyne-Stokes breathing J. Appl. Physiol. 20328

Norman R G, Goldring R M, Clain J M, Oppenheimer B W, Charney A N, Rapoport D M and Berger K I 2006 Transition from acute to chronic hypercapnia in patients with periodic breathing: predictions from a computer model J. Appl. Physiol. 1001733

Oliveira A J, Nunes M L, Fojo-Olmos A, Reis F M and da Costa J C 2004 Clinical correlates of periodic breathing in neonatal polysomnography Clin. Neurophysiol. 115 2247-51

Pereira M, Reis F, Landriault L, Cates D and Rigatto H 1995 Profile of alveolar gases during periodic and regular breathing in preterm infants Neonatology 67 322-9

Poets C F 2010 Apnea of prematurity: what can observational studies tell us about pathophysiology? Sleep Med. 11 701-7

Poets C F and Southall D P 1991 Patterns of oxygenation during periodic breathing in preterm infants Early Hum. Dev. 26 1-12

Rapoport D M, Norman R G and Goldring R M $1993 \mathrm{CO}_{2}$ homeostasis during periodic breathing: predictions from a computer model J. Appl. Physiol. 752302

Razi N M, DeLauter M and Pandit P B 2002 Periodic breathing and oxygen saturation in preterm infants at discharge J. Perinatol.: Official J. Calif. Perinat. Assoc. 22 442-4

Richerson G B and Boron W F 2005 Control of ventilation Medical Physiology ed W F Boron and E L Boulpaep (London: Elsevier Health Sciences) p 716 
Rigatto H 2003 Periodic breathing Respiratory Controls and Disorders in the Newborn ed O P Mathew (Boca Raton, FL: CRC Press) p 266

Simakajornboon N, Beckerman R C, Mack C, Sharon D and Gozal D 2002 Effect of supplemental oxygen on sleep architecture and cardiorespiratory events in preterm infants Pediatrics $110884-8$

Takahashi E and Doi K 1993 Destabilization of the respiratory control by hypoxic ventilatory depressions: a model analysis Japan. J. Physiol. 43 599-612

Tehrani F T 1997 A model study of periodic breathing, stability of the neonatal respiratory system, and causes of sudden infant death syndrome Med. Eng. Phys. 19 547-55

Verma V K, Katiyar V K and Singh M P 2009 Study of periodic breathing and human respiratory system Int. J. Med. Med. Sci. $1330-3$

Vielle B and Chauvet G 1998 Delay equation analysis of human respiratory stability Math. Biosci. 152 105-22

Vielle B and Chauvet G 1993a Cyclic model of respiration applied to asymmetrical ventilation and periodic breathing J. Biomed. Eng. 15 251-6

Vielle B and Chauvet G 1993b Mathematical study of periodic breathing as an instability of the respiratory system Math. Biosci. 114 149-72

Weintraub Z, Alvaro R, Kwiatkowski K, Cates D and Rigatto H 1992 Effects of inhaled oxygen (up to $40 \%$ ) on periodic breathing and apnea in preterm infants J. Appl. Physiol. 72 116-20

Wijdicks E F 2007 Biot's breathing J. Neurol. Neurosurg. Psychiatry 78 512-3

Wilkinson M H, Skuza E M, Rennie G C, Sands S A, Yiallourou S R, Horne R S and Berger P J 2007 Postnatal development of periodic breathing cycle duration in term and preterm infants Pediatr. Res. 62 331-6

Wilkinson M, Cranage S, Berger P, Blanch N and Adamson T 1995 Changes in the temporal structure of periodic breathing with postnatal development in preterm infants Pediatr. Res. 38 533-8 